\title{
Los acuerdos comerciales bilaterales: el caso colombiano (1990-2015)*
}

\author{
Bilateral Trade Agreements: the Colombian \\ Case (1990-2015)
}

\section{Acordos comerciais bilaterais: o caso colombiano (1990-2015)}

\author{
Carolina Rojas Sánchez ** \\ Álvaro Pío Gómez Olaya ${ }^{* * *}$
}

DOI: https://doi.org/10.19053/01203053.v37.n65.2018.5961

Fecha de recepción: 29 de marzo de 2017

Fecha de aprobación: 16 de noviembre de 2017

* Este artículo es el resultado de la investigación cofinanciada por la Universidad del Valle y Colciencias en el programa Jóvenes Investigadores de Colciencias. Trabajo de investigación adscrito al grupo"Gestión y Evaluación de Programas y Proyectos GYEPRO, Universidad del Valle.

** Economista y estudiante de Maestría en Estadística Universidad del Valle. Cali, Colombia. Dirección postal: Calle 4B No. 36-00 sede San Fernando, A.A. 25360 Correo electrónico: carojasanchez@gmail.com. (D) https:// orcid.org/0000-0003-1158-1456 Dirección postal: Calle 4B No. 36-00 sede San Fernando, A.A. 25360

*** Economista, Doctorado en Ciencias Económicas y Empresariales, Universidad Complutense de Madrid. Profesor del Departamento de Administración y Organizaciones Universidad del Valle. Cali, Colombia. Correo electrónico: alvaropiogomez@gmail.com. (D) https://orcid.org/0000-0002-2287-1646 


\section{Resumen}

La proliferación de acuerdos comerciales bilaterales ha creado un fenómeno conocido en la literatura como "organización regional en ejes" o bilateralismo "eje-radios" (hub-spoke). En este contexto, la política comercial de Colombia está orientada hacia la firma de acuerdos comerciales bilaterales. El artículo evalúa la política de firma de acuerdos bilaterales implementada por Colombia entre 1990-2015. Se analizan los flujos comerciales entre Colombia y diversos países aplicando el índice HM de Baldwin (2004) y la matriz de Chen (2008), que permiten medir la organización regional del comercio en ejes. Se encuentra que se ha consolidado un sistema de economías ejes y radios. Los resultados obtenidos cuestionan la política de firma de acuerdos colombiana.

Palabras clave: acuerdos de libre comercio (TLC), evaluación de política comercial, bilateralismo "eje-radios", integración regional, Colombia, Estados Unidos, China.

Clasificación JEL: F13, F14, F15. 


\section{Abstract}

The proliferation of bilateral trade agreements has created a phenomenon known in the literature as "hub and spoke" bilateralism. In this context, Colombia's trade policy is geared towards the signing of bilateral trade agreements. This article evaluates the policy of signing bilateral agreements implemented by Colombia between 1990 and 2015. We analyse trade flows between Colombia and several countries using Baldwin's HM index of hub-ness (2004) and the matrix of Chen (2008). The results obtained question the Colombian policy of signing trade agreements.

Keywords: free trade agreements, trade policy evaluation, hub and spoke bilateralism, regional integration, Colombia, United States of America, China 


\section{Resumo}

A proliferação de acordos comerciais bilaterais criou um fenômeno conhecido na literatura como "organização regional em eixos" ou bilateralismo "eixo-rádios" (hub-spoke). Neste contexto, a política comercial da Colômbia está orientada para a assinatura de acordos comerciais bilaterais. $\mathrm{O}$ artigo avalia a política de assinatura de acordos bilaterais implementados pela Colômbia entre 1990-2015. Os fluxos comerciais entre a Colômbia e vários países são analisados aplicando o índice HM da Baldwin (2004) e a matriz da Chen (2008) que permitem medir a organização regional do comércio em eixos, e verificou-se que um sistema de economias foi consolidado e rádios. Os resultados obtidos questionam a política de assinatura de acordos colombianos.

Palavras chave: Acordos comerciais bilaterais, Avaliação da política comercial, bilateralismo de "eixos-radios", Integração regional, Colômbia, Estados Unidos, China. 


\section{INTRODUCCIÓN}

A comienzos de la década de los noventa, Colombia inició el proceso formal de apertura económica. Sin embargo, en materia de políticas de integración comercial, particularmente de adopción de acuerdos comerciales, los avances se dan desde mucho antes, aunque tenuemente. En 1969, Colombia pertenecía al Pacto Andino, actual Comunidad Andina de Naciones (CAN), y en 1981, a la Asociación Latinoamericana de Integración (ALADI). También durante este año, Colombia se unió al Acuerdo General sobre Aranceles Aduaneros y Comercio (siglas en inglés GATT), aún vigente. Durante 1966-1974, Colombia se adhirió a una serie de acuerdos de preferencias arancelarias con distintos países, en especial de Europa, y a un conjunto de acuerdos de alcance parcial, firmados en 1984, con algunos países de Centroamérica.

Entre 1991-1994 crecieron los esfuerzos por la integración comercial regional, se da el primer Tratado de Libre Comercio (TLC) entre Colombia, México y Venezuela en 1994, denominado G3, y en ese mismo año participa del proyecto Área de Libre Comercio de las Américas (ALCA). En enero de 1995 se creó la Organización Mundial del Comercio (OMC), poco tiempo después, en el mes de abril, Colombia se adhirió a este organismo multilateral y se comprometió a profundizar su política de liberalización de la economía. Estas negociaciones multilaterales entraron en crisis debido al cambio en los gobiernos de los países miembros, a la poca capacidad de fijar contenidos 
y a la velocidad de negociación de dichos acuerdos (Vallejo, 2006).

Como respuesta a estas dificultades y a excepciones en los acuerdos de la $\mathrm{OMC}^{1}$, se estimuló una nueva ola de negociaciones de acuerdos bilaterales. En el caso de Colombia se empezó a materializar desde 2006 (Chile), Triángulo del Norte (2007), EFTA y Canadá (2008), en el 2012 entra en vigencia el TLC con Estados Unidos, principal socio comercial, suscrito desde el 2006. Continúa con el TLC con la Unión Europea (2012), Corea y Costa Rica (2013) y la Alianza del Pacífico (2014), Israel y Panamá (2016); actualmente se adelantan negociaciones con Japón y Turquía.

Esta política comercial, que privilegia la firma de acuerdos bilaterales como instrumento para avanzar en integración, se ha extendido por muchos países y ha generado una red de acuerdos que estructuran un sistema complejo, conocido en la literatura como: "bola de spagueti”. Según algunos estudios (Bhagwati, 1995; Wonnacott \& Wonnacott, 1995; Cornejo \& Harris, 2007; Menon, 2008), esta maraña de acuerdos, que incluso se traslapan, desarrolla un proceso caótico difícil de manejar, que cuestiona si efectivamente el acuerdo genera crecimiento, debido a costos administrativos y a posible incompatibilidad entre ellos.

Dentro de los fenómenos que generaron la proliferación de acuerdos, uno de los conceptualmente más sobresalientes fue planteado por Wonnacott y Wonnacott (1995), conocido como bilateralismo "hub-spoke" o sistema de integración eje-radios, y hace referencia a la manera como un país $\mathrm{A}$ (eje) al tener acceso al mercado de varios países (radiales) $-\mathrm{B}, \mathrm{C}, \ldots \mathrm{N}-$, sin que estos tengan acuerdos entre sí, puede disfrutar de ventajas al ser un mercado más atractivo para otros países, porque el país eje tiene preferencia en los mercados radios y los países radios enfrentan discriminación en los mercados radios y competencia en el mercado del país eje.

En este sentido, el presente artículo evalúa para el caso de Colombia, la política comercial de los gobiernos de este país, en el periodo 1990-2015, de negociación y suscripción de un

\footnotetext{
${ }^{1}$ La OMC ha señalado que los países no pueden normalmente establecer discriminaciones entre sus diversos interlocutores comerciales. Si se concede a un país una ventaja especial, se tiene que hacer lo mismo con todos los demás miembros de la OMC. Este principio se conoce como el trato de la nación más favorecida (NMF). Tiene tanta importancia, que es el primer artículo del GATT, que regula el comercio de mercancías. Sin embargo, se permiten ciertas excepciones. Por ejemplo, los países pueden establecer un Acuerdo de Libre Comercio que se aplique únicamente a los productos objeto de comercio dentro del grupo y hacer discriminaciones con respecto a los productos de terceros países. O pueden otorgar acceso especial a sus mercados a los países en desarrollo. O bien un país puede poner obstáculos a los productos que se consideren objeto de un comercio desleal procedentes de países específicos. Y, en el caso de los servicios, se permite que los países, en ciertas circunstancias restringidas, apliquen discriminaciones. Sin embargo, los acuerdos solo permiten estas excepciones con arreglo a condiciones estrictas (OMC, 2017).
} 
paquete de acuerdos comerciales bilaterales en el contexto eje-radios. Algunos estudios previos (Chen \& Lombaerde, 2011) dan indicios de que Colombia es una economía radio de Estados Unidos y, en este sentido, es relevante preguntarse si la política comercial de suscripción de acuerdos ha mejorado su condición de economía radio. En el mejor de los casos, ¿ha permitido la política comercial que Colombia adquiera potencial para convertirse en candidato a eje comercial para algún país o conjunto de países? Más allá ¿ha logrado que Colombia gane protagonismo en el escenario del comercio mundial?

Este artículo se divide en seis secciones. En la primera sección hay una reseña de la política comercial implementada en Colombia en los últimos años. La segunda sección presenta los estudios sobre el fenómeno hub-spoke y brinda algunas bases teóricas y resultados empíricos. En la tercera sección se explica la metodología empleada. La cuarta sección muestra los resultados del índice aplicado. En la quinta sección se desarrollan las implicaciones o efectos del fenómeno eje-radios para Colombia. En último término están las conclusiones.

\section{Política comercial e integración en Colombia 1990-2015}

El proceso de apertura económica en Colombia tiene su origen en el Gobierno de Virgilio Barco (1986-1990), que propuso un programa gradual de liberalización que combinaba una disminución lenta de las restricciones cuantitativas al comercio con medidas de protección a la industria nacional.

Sin embargo, es durante el Gobierno del presidente Gaviria (1990-1994) cuando se consolida la política de apertura económica y se produce un cambio sustancial en la orientación de la política de apertura pasando de ser gradual a ser aplicada de manera acelerada; se ejecuta un cronograma de liberalización comercial rápido, en el que se propone, por ejemplo, la eliminación total de las licencias previas de importación durante el primer año de gobierno, una reducción del nivel promedio de aranceles que debía pasar de un nivel promedio de $34,6 \%$ a un nivel promedio de $15 \%$ al final del periodo de gobierno ${ }^{2}$.

La reforma comercial del Gobierno Gaviria (1990-1994), en materia de integración económica, originó la firma de acuerdos de alcance parcial (AAP)

\footnotetext{
$\overline{2}$ Ver Guacaneme (2011) para una mayor ampliación sobre los criterios establecidos por el CONPES para el proceso de internacionalización de la economía colombiana.
} 
con varios países de la región. En este periodo se crea el AAP con Argentina, Bolivia, Brasil, Chile, Cuba, Ecuador, Paraguay, Perú, Panamá y Bulgaria. Entra en vigencia en 1992 la Ley de Preferencias Arancelarias Andinas (ATPA) con los Estados Unidos. En 1994 se da el primer TLC del país, con México y Venezuela. En este año se adhiere a la Organización Mundial de Comercio OMC. Por otro lado, se continúan impulsando los procesos vigentes: las negociaciones del ALCA, fortalecimiento del proceso de integración a través de la CAN y participación en acuerdos de preferencias arancelarias (SGP) con algunos países de Europa.

La administración del presidente Samper (1994-1998) le da continuidad a la política de integración, privilegiando los procesos de conformación de bloques comerciales. En su Plan Nacional de Desarrollo (PND) "El Salto Social" propone que Colombia sea un actor activo de los procesos de integración económica en América Latina. De esta manera, se apoya el proceso de consolidación del Grupo Andino y la Asociación de Estados del Caribe, y la ampliación de la agenda de negociaciones con los países del Mercado Común Centroamericano y se da inicio al proceso con MERCOSUR. En el caso del Grupo Andino se propone una integración que alcance la armonización gradual de políticas agropecuarias y macroeconómicas.
Por su parte, el Gobierno de Pastrana (1998-2002) buscó posicionar las exportaciones como motor de desarrollo para afrontar la profunda crisis política y económica que atravesaba el país. El Gobierno formuló un proyecto denominado: "Plan estratégico Exportador 1990-2009", con el cual pretendió lograr el fortalecimiento del sector productivo colombiano y su orientación al mercado internacional. El plan exportador adoptado permitió aumentar las exportaciones y diversificar la oferta en productos y mercados en función de la demanda mundial. Por estos resultados positivos, el plan estratégico exportador tendrá continuidad en el próximo Gobierno.

En medio de la crisis, se marcó más la dependencia con Estados Unidos tanto en la lucha contra las drogas como en lo económico. Por eso, la política de comercio exterior propuso "mirar hacia el norte" (Cardona, 2001). De allí que un logro para este Gobierno fue alcanzar la prórroga del ATPA (vencida en 2001) y renovada hasta el 2005 como ATPDEA. También se adelantaron acercamientos con otros países. En 2000 se firma el Acuerdo de Alcance Parcial de Complementación (AAPC) en Cuba. Y en 2002, un acuerdo de preferencias otorgado por Bulgaria y otro por Turquía. Por otra parte, las negociaciones multilaterales (principalmente el ALCA) entran en una etapa de estancamiento. 
Tal y como lo plantea Guacaneme (2011), los gobiernos a lo largo de la década de los noventa profundizan el proceso de apertura económica y consolidan el modelo económico; si bien cambiando el estilo y los instrumentos, la política sigue vigente. De esta manera, se podría afirmar que el modelo de integración es una política de Estado:

Y así se refleja en los distintos Planes de Gobierno de los Presidentes electos a lo largo de la Década. Al estudiar la política comercial de cada uno de ellos, es claro que con los matices propios que cada uno quiere imprimir o con la prioridad que cada uno le da al tema, siempre está presente la continuación de un proceso de apertura económica, ya sea en el Plan El salto social (1994) de Ernesto Samper Pizano, Capítulo Agenda Internacional, o en el de Andrés Pastrana Arango, Cambio para construir la paz (1998). (Guacaneme, 2011, p. 7)

En el primer periodo del Gobierno de Uribe (2002-2006), la política comercial se asociaba con el fácil acceso a los mercados externos y a la armonización de las normas que regulan la actividad económica. El PND propuso profundizar las relaciones bilaterales estratégicas con Estados Unidos, la Unión Europea (EU), MERCOSUR y la región de Asia-Pacífico. En este Gobierno se busca avanzar decididamente hacia la integración comercial. El PND propone una política comercial enfocada en "la negociación de acuerdos internacionales que eliminen barreras a acceso, estimulen la inversión extranjera $\mathrm{y}$ faciliten la reasignación de recursos en actividades productivas" (PND Gobierno Uribe 2002-2006, p. 134). En materia de logros, entra en vigencia el AAPC CAN-MERCOSUR en el 2004 y comienzan las negociaciones de un TLC con Chile. De esta manera, se propone, al igual que los gobiernos anteriores, continuar y profundizar la apertura económica.

El presidente Uribe en su segundo mandato (2006-2010) consolida la idea de lograr el desarrollo profundizando la integración con la economía mundial y que, por lo tanto, es necesaria la suscripción de acuerdos internacionales de comercio de última generación ${ }^{3}$. Continúa las negociaciones que se venían adelantando y, al mismo tiempo, se concretan otros acuerdos comerciales (Chile 2006, Triángulo del Norte 2007, EFTA y Canadá 2007).

Pero, sin lugar a dudas, una de las prioridades en el tema comercial de los dos periodos de Gobierno Uribe fue el TLC con Estados Unidos. Sin

\footnotetext{
${ }^{3}$ Los acuerdos internacionales comerciales de última generación tienen un amplio contenido cualitativo, es decir, se tratan otros temas fuera de lo económico, como seguridad y democracia, cambios en el mercado laboral, derechos de autor, inmigración, terrorismo, entre muchos otros.
} 
embargo, el proceso para firmar el TLC fue largo. Desde el 2004 empiezan las negociaciones, pasando por una etapa de congelamiento en el Congreso de los Estados Unidos, que impidió la firma del TLC durante la administración de Uribe. El Gobierno de Estados Unidos da una prórroga del ATPDEA hasta que llegue el TLC.

Por otra parte, la CAN atravesaba una de sus peores crisis. Uno de los hechos que más influyó fue el retiro de Venezuela en el 2006, país que arguyó que las negociaciones del TLC de Estados Unidos con Perú y Colombia iban en contravía de lo ambicionado para la CAN.

De igual forma en el 2006, el Gobierno venezolano se retiró del G3. Esta vez argumentando competencia desleal, pérdidas de la productividad y empleo, y señalando su decisión de fortalecer la integración con MERCOSUR. De ahí que los flujos comerciales entre ambos países se vieron afectados, lo cual implicó, al menos para Colombia, distanciarse de un importante socio comercial. Esto sumado a los problemas políticos de los gobiernos de Chávez y de Uribe.

En 2010 asume el mandato el presidente Santos. En su PND, en materia de comercio exterior, plantea que una de las estrategias para mejorar el acceso de los productos colombianos al exterior es "avanzar en la negociación e implementación de acuerdos comerciales en concordancia con las oportunidades de mercado identificadas en las agendas (PND Gobierno Santos 2010-2014, 239)". El PND estableció una estrategia de política arancelaria ligada a la "transformación productiva.

El Gobierno logra en el 2011 mejorar sus relaciones con Venezuela firmando un AAP. En ese mismo año, se firma el TLC con Canadá. Análogamente, consolidando los esfuerzos del Gobierno anterior, entra en vigencia el TLC con Estados Unidos. Además se negocian y suscriben varios TLC ${ }^{4}$.

El segundo mandato del presidente Santos (2014-2018) ha seguido los lineamientos del primero, marcado por una fuerte dependencia de las exportaciones relacionadas con el sector minero-energético y los intentos por diversificar el portafolio de socios comerciales. El PND 2014-2018 plantea al respecto que uno de los resultados que mostrará una evolución favorable en el desarrollo productivo e internacionalización del país será el incremento de las exportaciones de bienes no minero-energéticos y de servicios.

En síntesis, desde el año 1990, los distintos gobiernos colombianos han

\footnotetext{
$\overline{{ }^{4} \text { Hacen parte }}$ de esta lista: Costa Rica, Corea del Sur, Turquía, Panamá Israel y la denominada Alianza Pacífico.
} 
orientado su política comercial hacia la profundización del proceso de apertura comercial. Si bien los gobiernos han hecho énfasis en diversos instrumentos de política, puede constatarse una continuidad en la política de integración comercial, la cual ha privilegiado el uso de acuerdos comerciales bilaterales como instrumento de política, especialmente en el período comprendido entre el año 2002 (en el primer Gobierno de Uribe) y el año 2015.

\section{Revisión de la literatura sobre el fenómeno eje-radios (hub-spoke)}

En la literatura, el término hub-spoke o "eje-radios" ha sido tratado ampliamente (Kowalczyk \& Wonnacott, 1992; Baldwin, 1995; Wonnacott, 1996; De Santis, De Benedictis \& Vicarelli, 2005; Deltas, Desmet \& Facchini, 2006, 2012; Alba, Jung \& Park, 2010). Sin embargo es importante empezar por su definición. En esencia, el bilateralismo eje-radios se genera cuando existen al menos tres países $\mathrm{A}, \mathrm{B}$ y $\mathrm{C}$; A y B tienen un acuerdo de libre comercio. Luego, A firma un nuevo acuerdo con el país C. El país A se denomina eje y los países $\mathrm{B}$ y $\mathrm{C}$ se denominarían radios. El país A gozaría del acceso preferencial a los mercados $\mathrm{B}$ y $\mathrm{C}$, mientras que $\mathrm{B}$ y $\mathrm{C}$ competirían en el país A por una cuota de mercado. ¿Cuáles serían los resultados del patrón eje-radios? (Lee, Park \& Shin, 2005).
Los pioneros en analizar el efecto del bilateralismo eje-radios explican los siguientes problemas:

Las exportaciones del país eje tiene preferencias en los mercados radios y los países radios se enfrentan a la discriminación en los mercados radiales y competencia en el mercado del país eje. Asimismo, la localización de las industrias es más atractiva en el país eje que en los países radios ya que tiene acceso a ambos mercados radiales. A su vez, podría darse que algunas exportaciones entre los países radios sería redireccionada a través del país eje [...]. (Wonnacott \& Wonnacott 1995, p. 4)

Entonces ¿quién quiere ser una economía radio? Si los países radios identifican las desventajas de ser economía radio empezará una carrera por alcanzar el estatus de eje. Sin embargo, no todos los países pueden convertirse en eje ¿De qué depende ser o no un eje?

"Un eje será un país cuyo acceso preferencial es altamente valorado por la mayoría, o por todos los países en la región" (Baldwin, 2004, p. 27). En definitiva, dependerá del interés de los demás países para entrar en el mercado del país eje.

Los primeros estudios examinan implicaciones de política del sistema eje-radios para Canadá (Wonnacott \& Wonnacott, 1982; Wonnacott, 1996). También sobre efectos del bilatera- 
lismo eje-radios dentro del NAFTA (Kowalczyk \& Wonnacott, 1992; Wonnacott, 1996). En particular, el primer estudio (Kowalczyck \& Wonnacott, 1992) concluye que para los países miembros es más beneficioso ampliar el acuerdo regional que establecer un bilateralismo eje-radios, porque dicho sistema tiene asociados costos de transporte y de administración más altos.

Por otra parte, se han desarrollado investigaciones puramente empíricas de los efectos del bilateralismo eje-radios; en Europa (Martin \& Turrion, 2001; Laaser \& Schrader, 2002; Adam, Kosma \& McHugh, 2003; Paas, 2003), en Israel (Deltas et al., 2006) y en Singapur, Japón, y Estados Unidos (Chong $\&$ Hur, 2008).

Un trabajo base para el desarrollo es el de Baldwin (2004), quien presenta algunos apuntes teóricos sobre el fenómeno y realiza un análisis para los países del Este Asiático. El autor plantea un índice HM que permite medir la organización regional del comercio en ejes, para medir el interés de otras naciones de la región por entrar al mercado de un país; siendo el país valorado, un eje. El estudio determina que los dos grandes ejes de la región son Japón y China y, en menor medida, Corea. Del estudio se destacan dos conclusiones: en primer lugar, que el fenómeno eje-radios produce un resultado inferior para la región en su conjunto, y en segundo lugar, que una proliferación de acuerdos comerciales podría llevar a divisiones políticas.

El trabajo de Lee et al. (2008) estudia los efectos de múltiples acuerdos de libre comercio, su interacción y su evolución en el tiempo para 175 países entre 1948-1999, y encuentra que la creación de comercio es más baja si los países se hallan bajo una configuración eje-radios.

En Latinoamérica, los países que están en la carrera por alcanzar el calificativo de ejes son México y Brasil $^{5}$ (Wonnacott \& Wonnacott, 1995; Baldwin, 2004; Chen \& Lombaerde, 2011). En el caso de México, el país ha quitado unilateralmente sus barreras no arancelarias y arancelarias en algunos sectores, ha suscrito varios acuerdos bilaterales, en especial con Estados Unidos y Canadá, y ha realizado algunas reformas internas para atraer la inversión extranjera directa. Por otra parte, Brasil tiene una agenda bilateral menos dinámica, pero apoya fuertemente el proceso de integración regional a través de MERCOSUR.

\footnotetext{
${ }^{5}$ Según información de la Organización Mundial del Comercio (OMC), México por ejemplo tiene acuerdos comerciales con Estados Unidos, Canadá, Colombia, Venezuela, Bolivia, Chile, Costa Rica, Nicaragua, Honduras, Guatemala, El Salvador, y Uruguay.
} 
Chen y Lombaerde (2011) analizan el fenómeno en América latina y el Caribe, se destaca la importancia del papel de Estados Unidos como eje de la región. Sin embargo, no desconocen los roles de México y Brasil, que muestran dos modelos de desarrollo comercial diferentes. México, apadrinado por Estados Unidos y Brasil, es más independiente en la industrialización de su economía doméstica. De esta forma, Brasil puede ser un candidato a convertirse en eje del Cono Sur (en el marco del MERCOSUR) y México ha empezado a tener importancia para los países de Centroamérica, pero débilmente.

Para el caso colombiano, los estudios sobre integración comercial, generalmente, se han enfocado en evaluar la efectividad de los acuerdos comerciales vigentes considerándolos individualmente y no el paquete de acuerdos firmados. Entre ellos se encuentran los estudios sobre efectos de la CAN y el MERCOSUR (Carrillo \& Li 2002); relación entre la CAN y el sistema preferencial andino que dan los Estados Unidos (Vallejo, 2006); eficiencia del Sistema de Preferencias Arancelarias de la UE (SGPUE) para promover las exportaciones colombianas (Correia, 2008).

Un estudio colombiano, de gran importancia para esta evaluación, plantea que el modelo general en América es el denominado "eje-centro", siendo
Estados Unidos el "país eje" que se erige sobre un sistema hemisférico de múltiples "radios" de TLC de nueva generación. Los autores concluyen que la política comercial colombiana se adhiere al modelo del regionalismo abierto dentro del sistema eje-rayos con Estados Unidos como eje principal en el campo comercial y hegemónico político del hemisferio (Lombaerde \& Garay 2009).

La revisión anterior muestra que los estudios que exponen el caso colombiano bajo el enfoque de bilateralismo eje-radios son escasos y tratan a Colombia tangencialmente cuando analizan el fenómeno para América (Baldwin, 2004; Chen \& Lombaerde, 2011). Por lo tanto, el presente artículo plantea una evaluación de la política de firma de acuerdos para el caso colombiano en el contexto eje-radios, desde la apertura comercial hasta el 2015, mostrando la evolución de los flujos comerciales y comparando entre países, mediante la combinación del índice HM y la matriz de Chen.

\section{METODOLOGÍA}

Para estudiar el fenómeno eje-radios para el caso colombiano inicialmente se debe poder identificar el papel que tiene cada país dentro de esta estructura, es decir, determinar quién se considera eje o no de su socio comercial. El propósito es poder identificar si Colombia ha ganado protagonismo en el 
mercado de sus socios, convirtiéndose en eje o si, por el contrario, es una economía radio.

Con este fin, se plantean dos aspectos metodológicos relevantes:

El índice HM, el cual mide la organización regional del comercio en un esquema radial, es decir, la capacidad que tiene un país de atraer el interés comercial de las demás economías (Baldwin, 2004). En otras palabras, mide el interés de todas las economías por entrar al mercado de ese país. Por ello, las economías buscarán firmar acuerdos bilaterales con este país - lo cual lo convertirá en eje - porque les permite un acceso preferencial a su mercado interno, lo que se verá representado en unas mayores exportaciones.

El índice HM permitirá entonces determinar qué país es eje o no. La medida es útil porque posibilita determinar si Colombia puede ser eje o no para algún país o sistema radial de países.

Usando la información de la División de Estadísticas de Comercio Internacional de la ONU (UN Comtrade, 2017) se calcula el índice HM para aproximadamente 160 países socios de Colombia durante 19902015. Es importante analizar este periodo, porque abarca el proceso de apertura comercial de Colombia en los primeros años de los noventa, el estancamiento de los acuerdos multilaterales y la transición al fortalecimiento de la política comercial de firma de acuerdos comerciales en los últimos años.

La medida se estima diferenciando tres casos distintos, de la siguiente manera:

- Si Colombia es eje o candidato a serlo. El índice HM corresponde a la evolución temporal de la atracción o influencia del mercado colombiano desde la perspectiva de sus socios comerciales, es decir, si Colombia se considera eje o candidato a serlo para un país o varios de ellos. Los resultados presentados corresponden a los primeros diez países con el índice más alto.

- Si Colombia es una economía radio. El índice HM corresponde a la evolución anual de la atracción o influencia del país socio comercial desde la perspectiva de Colombia, es decir, el índice HM determinará qué economía es eje para Colombia. Los resultados presentados corresponden a los primeros diez países hubs para Colombia, los que tengan el índice más alto.

- Colombia frente a otros países radios. El índice HM se calcula para cada pareja de países por quinquenios en los años 1990, 1995, 2000, 2005,2010 y 2015. Se trata entonces de un análisis comparativo, en 
donde se toma una "fotografía" cada cinco años para medir la evolución de Colombia con respecto a otros países del hemisferio occidental. El análisis consiste en elegir un eje (la elección se explicará más adelante en la matriz HM) y comparar la evolución de Colombia con los demás países de América en cada uno de los años. El periodo de tiempo no se toma como continuo por facilitar el análisis. El lapso de cinco años fue seleccionado porque se busca un análisis muy preciso para mostrar los cambios de manera detallada pero no de forma tan inmediata que no pudieran ser percibidos. Los resultados son analizados para los países de América divididos en tres subregiones: norte, centro y sur.

A continuación se explica un poco la idea básica de la fórmula para el cálculo del índice HM y su interpretación.

A partir de la ecuación gravitacional aplicada al modelo de comercio de Helpman-Krugman (1985) bajo el enfoque de competición monopolística de Dixit-Stiglitz ${ }^{6}$, Baldwin deriva una medida que permite identificar la organización del esquema eje-radios, es decir, quién es eje $y$, por ende, quién es radio.
La idea básica del índice HM es medir si el acceso preferencial al mercado de un país es valorado por otros países. El índice HM supone que el interés de los exportadores es proporcional a las exportaciones extra que la apertura bilateral ofrecerá. Conforme a esto, Baldwin busca determinar cuánto aumentan las exportaciones del país i si obtiene acceso preferencial a otro país j y cuál es el incremento en el total de las exportaciones del país i. De acuerdo con lo anterior se plantea la ecuación 1 :

$$
X_{i}=X_{i a j}+\sum_{m} X_{i a m}
$$

Donde $X_{i}$ son las exportaciones totales del país i, $X_{i a j}$ son las exportaciones del país i con destino a j y $\sum_{m} x_{i a m}$ son las exportaciones de i a otros países. En una diferenciación logarítmica se muestra que el incremento porcentual de las exportaciones totales que se deriva de un incremento del $1 \%$ de $X_{i a j}$ es la parte del país j en las exportaciones de i, $X_{i a j} / X_{i}$.

Por otro lado, Baldwin plantea la importancia de mirar cuál es el peso de las exportaciones de $\mathrm{i}$ hacia $\mathrm{j}$ sobre el total de importaciones de $\mathrm{j}$, una vez se obtiene acceso preferencial, es decir, cuando se reducen los aranceles (por la entrada en vigencia de un acuerdo

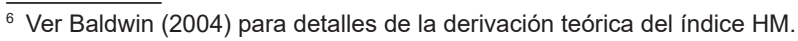


bilateral) cuánto aumentará lo que le compra el país $\mathrm{j}$ a $\mathrm{i}$ en el total de las importaciones de $\mathrm{j}$.

Uniendo las dos partes, el autor propone el índice HM (ecuación 2) como una estimación aproximada del incremento de las exportaciones de $\mathrm{i}$ a $\mathrm{j}$ que resultan de un cambio marginal en el acceso preferencial como:

$$
H M_{i}=s_{i j}^{X}\left(1-s_{i j}^{M}\right)
$$

Donde $H M_{i}$ mide la organización en ejes del país i, es decir, el atractivo comercial del país j desde el punto de vista del país i. $s_{i j}^{X}$ es la proporción de las exportaciones de i que van a $\mathrm{j}$ sobre el total de las exportaciones de i y $s_{i j}^{M}$ es la proporción de la importaciones de $\mathrm{j}$ que provienen de $\mathrm{i}$, sobre el total de las importaciones de j. $H M_{i}$ aumenta con la importancia de las exportaciones de i que van a $\mathrm{j}$, y disminuye con la importancia de la importaciones de $\mathrm{j}$ que vienen $\mathrm{i}$. El índice tomará valores entre 0 a 1 , entre más se acerque a 1 las exportaciones del país i dependerán más de $\mathrm{j}$ y por lo tanto $\mathrm{j}$ será un eje para $\mathrm{i}$.

En cuanto a la matriz HM, esta se construye con el propósito de obtener una clasificación o ranking del país o los países considerados ejes más importantes para ese conjunto de países (Chen, 2008). El cálculo de la matriz
HM se hace a partir de la estimación del índice HM, de tal forma que los resultados obtenidos para cada pareja de países se organicen en una matriz de nxn (denominada matriz HM), donde $n$ es el número de países en el análisis. En las columnas se encuentran los posibles ejes y en las filas los países radios. La diagonal de la matriz no tiene valores.

Después se toma cada país radio-fila y se compara por posibles ejes-columnas, realizando un ranking que toma el valor de 1 si el país es de mayor importancia para el otro - es decir, un HM mayor-y así sucesivamente de acuerdo con el número de países en el análisis, llegando hasta n. Posteriormente, por cada columna se suman los resultados y se obtiene un ranking total. El menor valor obtenido corresponde al país o países considerados ejes de la región.

Ahora bien, la matriz HM será usada para seleccionar los ejes más importantes en el mundo, con el fin de analizar el estado y evolución por quinquenios de Colombia y las demás economías radios del hemisferio con respecto a cada eje.

\section{Aplicación}

La aplicación está dividida en dos tipos de análisis: 
1. Un análisis temporal de la evolución de Colombia frente al sistema eje-radios durante 1990-2015, calculando el índice HM. A su vez, en este análisis se abordan dos casos:

a. Cuando Colombia se considera candidato a convertirse eje comercial para un país o conjunto de países radios y, b. Cuando Colombia es una economía radio.

2. Un análisis comparativo por país eje de Colombia frente a los demás países radios de América Latina por quinquenios desde $1990 \mathrm{a}$ 2015 usando la matriz HM de Chen (2008).

a) ¿Puede Colombia ser candidato a convertirse en eje comercial? Evolución de las principales economías radios de Colombia 19902015

A efectos de este análisis, se presentan únicamente los resultados de los primeros diez países con el mayor índice HM de Colombia en el periodo comprendido entre 1990-2015 (Figura 1). La principal conclusión es que la evolución volátil del índice HM y la magnitud en cada país no permite considerar a Colombia como candidato firme a convertirse en país eje para algún país radio o sistema radial de países. Dentro de los países involucrados se señala que todos están ubicados en el contexto regional (son de América Latina y el Caribe), resultado que tiene cierta lógica, dado que la economía colombiana es pequeña y tiene mayores posibilidades de influir en la región, con sus socios "naturales".

De igual forma, todos los países de la CAN se encuentran dentro de este conjunto. No obstante, aunque se esperaría que el índice representara una relación más estrecha y estable con respecto a estos países, debido a que es un proceso de integración que viene desde 1969 y que, en términos comparativos, en el interior de la CAN se considera a Colombia como una de las economías líderes en dicho proceso de integración, esto no ocurre; el índice es variable y su magnitud es pequeña, lo que indica que, incluso en el interior de la CAN, Colombia no representa un eje de los países miembros.

En este sentido, es importante indicar que en algún momento para los países andinos, Bolivia y Ecuador, los índices HM presentaban cifras superiores al $5 \%$ (1994-2003), pero estas fueron cayendo gradualmente. 


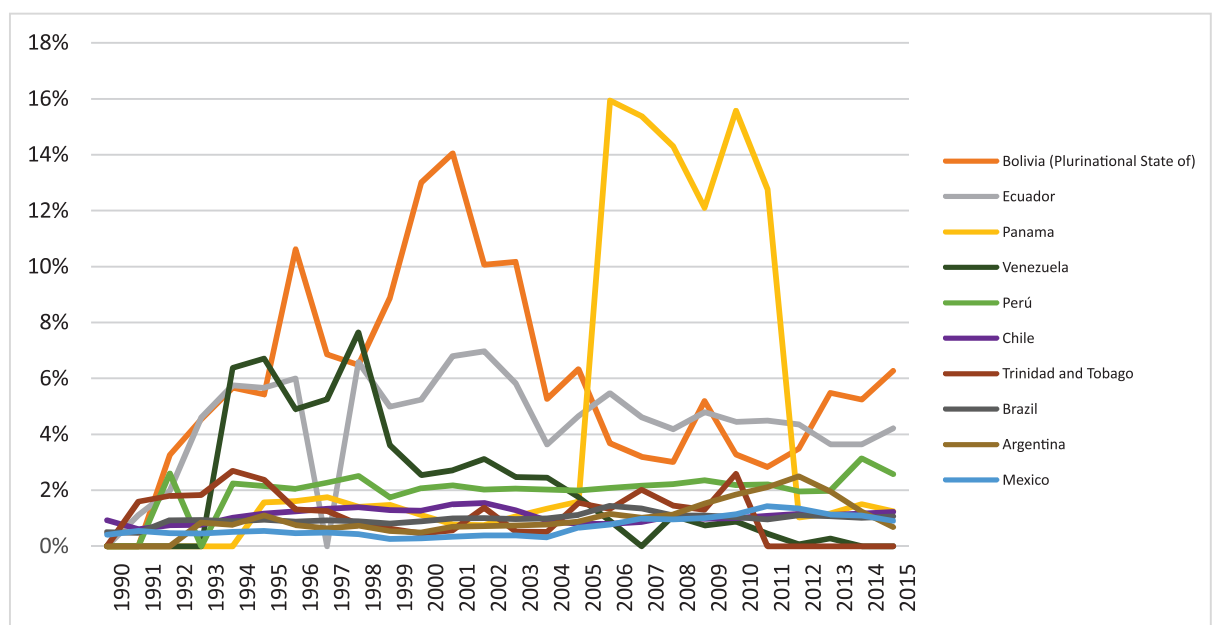

Figura 1. Evolución del índice HM de Colombia entre 1990-2015 para los primeros diez países.

Fuente: elaborado con base en datos de UNCOMTRADE (2017).

En síntesis, el cálculo permite determinar que Colombia no puede ser considerado candidato a convertirse en país eje comercial para algún país o conjunto de países en el periodo 19902015 (ver Figura 1). Si bien respecto de países como Bolivia, Ecuador y Panamá, logró ganar protagonismo en estos mercados, no pudo sostenerlo; en otras palabras, Colombia sufrió un retroceso en su carrera por convertirse en eje comercial en estos países, y aún en este caso, los avances no serían atribuibles a la política de firma de acuerdos comerciales bilaterales, sino a la dinámica de otro proceso de integración: la CAN.

\section{b) ¿Cuáles son las economías ejes para Colombia? Evolución de la dependencia comercial entre 1990-2015}

Se presentan únicamente los primeros diez países con los índices HM más altos, que son posibles economías ejes para Colombia entre 1990-2015 (ver Figura 2). En este caso, el índice HM muestra resultados contundentes: Estados Unidos es el país eje para Colombia. A lo largo del periodo de análisis, el índice HM se ubica en promedio en un 40 \%. En los años 1999 y 2000 alcanza incluso niveles por encima al $50 \%$, es decir, una dependencia considerable hacia este país. Aunque desde el año 2000 se ha presentado una reducción constante hasta el 2015, la magnitud del índice es alta. 


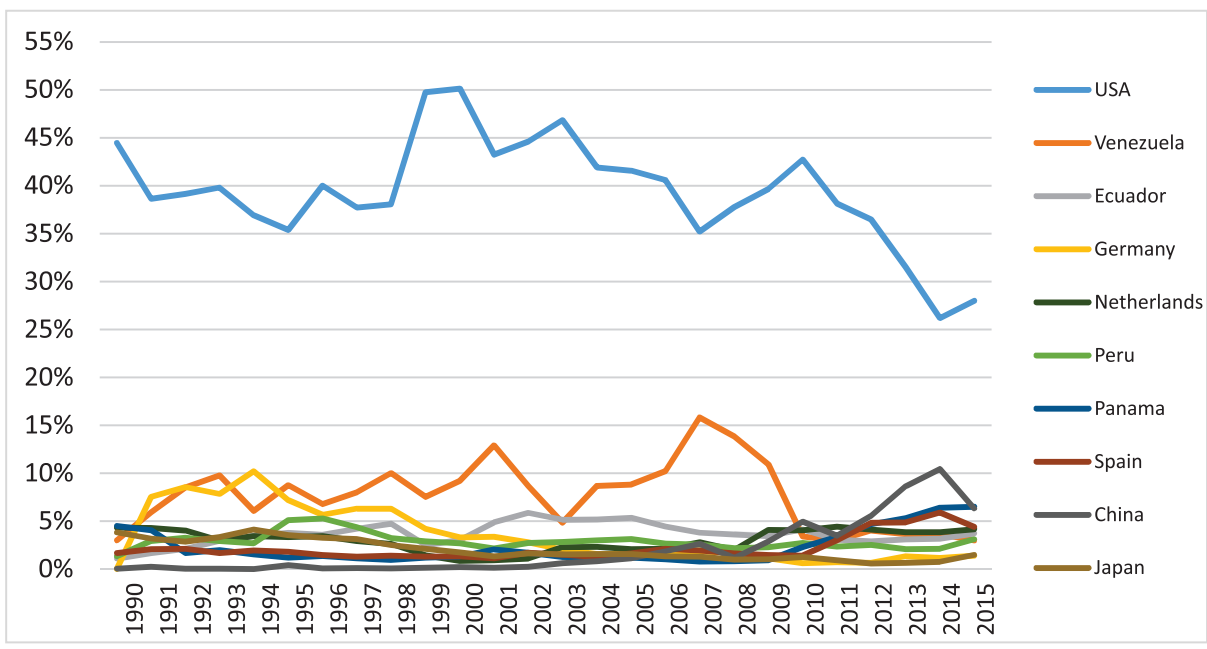

Figura 2. Evolución del índice HM para los diez principales ejes de Colombia entre 1990-2015.

Fuente: elaborado con base en datos de UNCOMTRADE (2017).

El segundo país respecto del cual Colombia presenta un grado importante de dependencia comercial es Venezuela, el cual, a pesar de haberse retirado de los dos acuerdos comerciales que sostenía con Colombia (la CAN y el G3), mantuvo los niveles del índice HM por encima del $5 \%$ en el periodo 1991-2009, alcanzando incluso un máximo de $16 \%$ en el año 2007. Es interesante ver como en el periodo de distanciamiento (2006-2009), el índice estaba en cifras entre 10 y $16 \%$ y después cayó de manera sostenida hasta el año 2015.

En el listado de 10 países, pertenecen a la región -Ecuador, Perú, Chile, Panamá, México y Brasil-, con excepción de dos países asiáticos: Japón y China. Se señala, que si bien estos países se encuentran dentro de los diez más altos valores del índice HM de Colombia, su participación es pequeña y todavía no se consideran economías potencialmente ejes para Colombia; no obstante es llamativo el avance alcanzado por la economía China, con una dinámica llamativa desde al año 2010 en el cual alcanzó valores cercanos al $5 \%$, los cuales se situaron alrededor del $10 \%$ en 2014.

\section{c) Comparación por quinquenios de Colombia frente a las demás economías radios del hemisferio: resultados por país eje}

Inicialmente, para realizar el análisis comparativo entre economías 
radiales de América, como se explicó en la metodología, se construye la matriz HM para el nivel mundial (Chen 2008). El ranking se obtiene para 119 países del mundo, de los cuales se extraen los diez primeros, a fin de seleccionar los más grandes ejes para cada quinquenio de 1990 hasta 2015. Se muestra la posición de los países ejes más importantes del mundo y su evolución por quinquenios (cuál baja y cuál sube), y con fines puramente ilustrativos la posición de Colombia (ver Tabla 1).

Estados Unidos es el país-eje más importante del mundo en todo el periodo estudiado. A partir de 1995 empieza a aparecer Alemania en los primeros lugares. Otras economías ejes son países europeos miembros de la Unión Europea, siendo preponderantes el Reino Unido y Francia. De igual forma, se encuentran únicamente dos países del Este Asiático: Japón y China. Es sumamente importante destacar el caso de China, que en los últimos 25 años pasó de no figurar en el ranking a estar en el séptimo lugar en 2005 , en el 2010 al quinto lugar, y en el 2015 llegó ser el segundo eje más grande en el mundo. En contraste, otra potencia económica asiática, Japón, ha perdido posiciones como eje desde 1990. A principios de la década los noventa, se ubicaba en el quinto lugar de los países ejes mundiales. Ya para el año 2000 había perdido cuatro posiciones, ubicándose en el noveno lugar. En 2005 es el último año en que Japón entra en el grupo de los diez principales. Al parecer, en 2010 y 2015 dicha posición ha sido ganada por India, que se localizó en el décimo puesto en los últimos dos quinquenios analizados. 
Apuntes CENES Volumen 37, Número 65

enero - junio 2018. Págs. 117-149

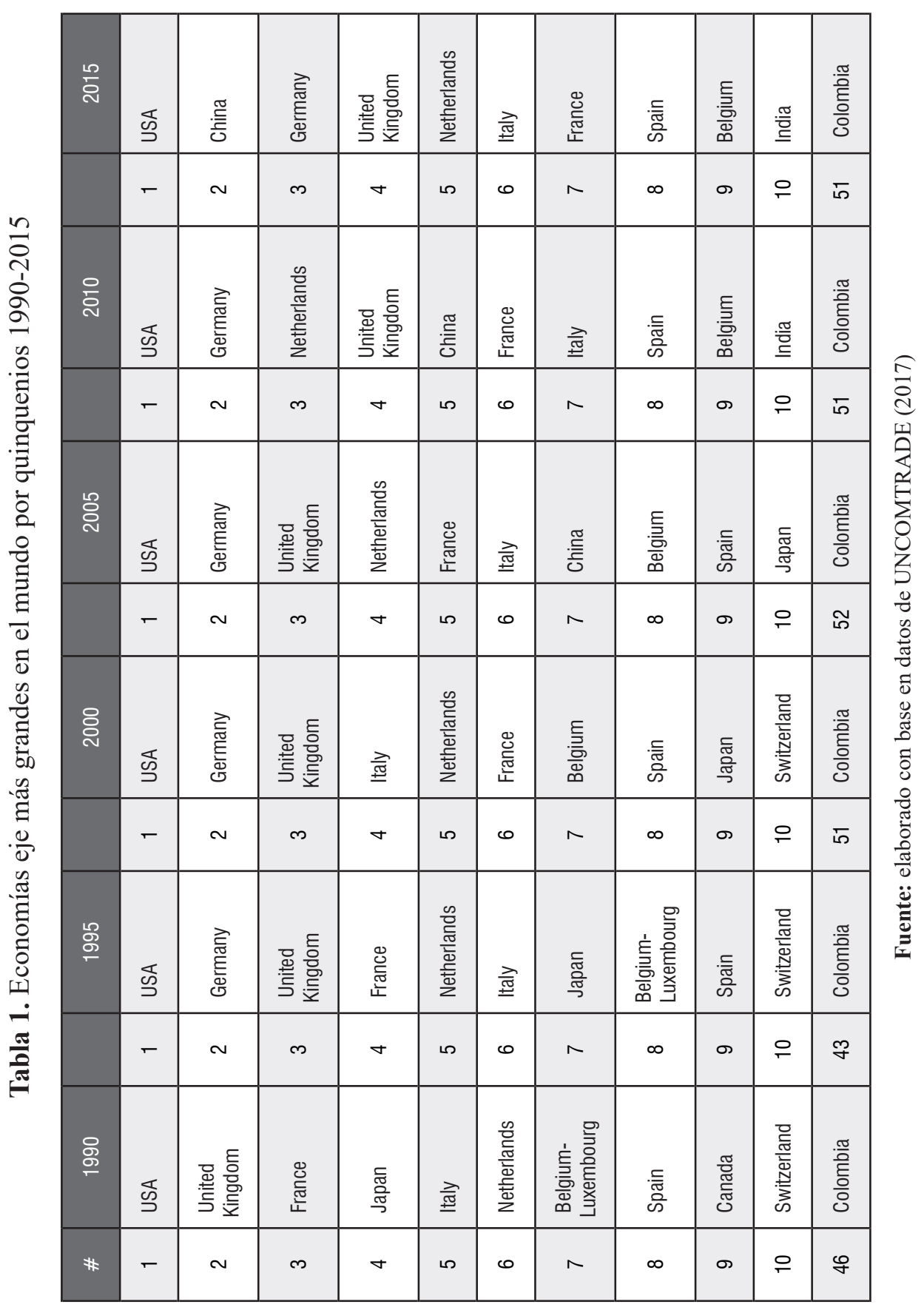


Con respecto a las grandes economías eje identificadas en el mundo, Estados Unidos, los países europeos miembros de la Unión Europea, Japón y China; se compara el estado y evolución de Colombia y los países radios de norte, centro y sur de América en estos años específicos.

\section{d) Estados Unidos, "el gran eje"}

Para evaluar el rol de los Estados Unidos como economía eje en la región, se presentan los valores del índice HM para el sistema radial de países de América con relación al "gran eje Estados Unidos” (ver Figura 3). En la parte izquierda de la gráfica están los países del norte y centro y en la parte derecha los países radio de Suramérica. En primer lugar, México y Canadá, que tienen un acuerdo comercial con Estados Unidos, el NAFTA, presentan un HM mayor (alcanzando incluso un $84 \%$ en el periodo de análisis), es decir, tienen una mayor dependencia comercial con este eje.

En Centroamérica se muestran porcentajes muy variados, pero que, en general, son altos para todos los países estudiados, superiores al $40 \%$ en casi todos los casos. Aunque se evidencia una tendencia descendente para algunos países.

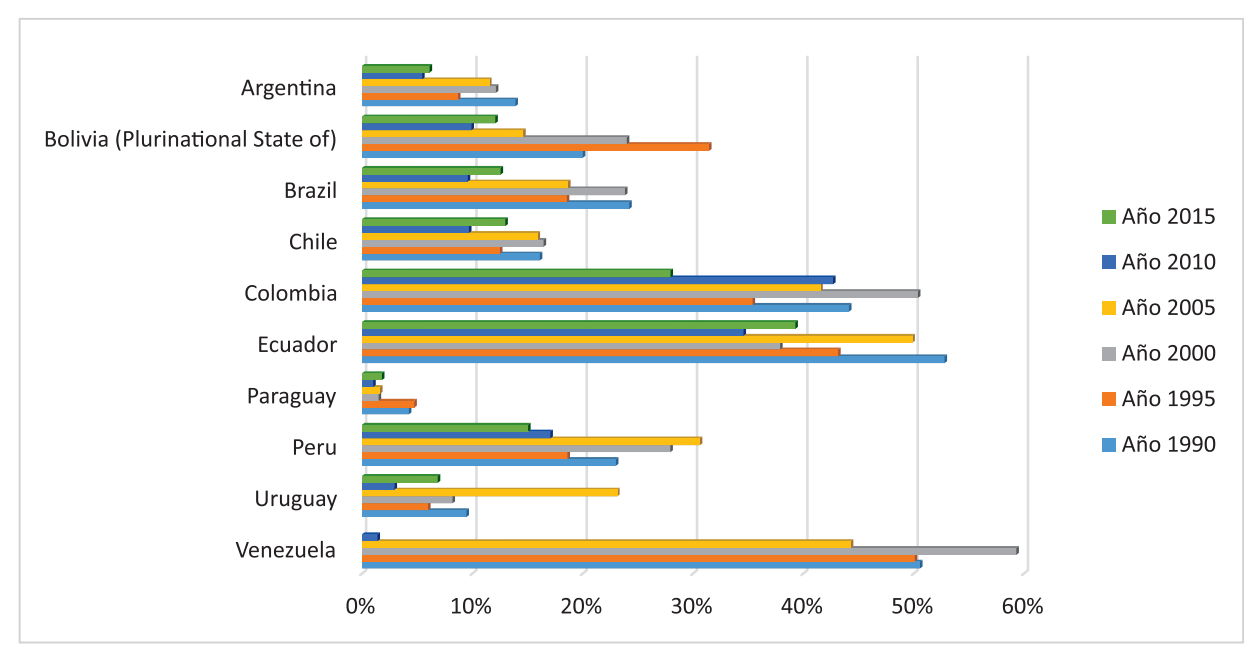




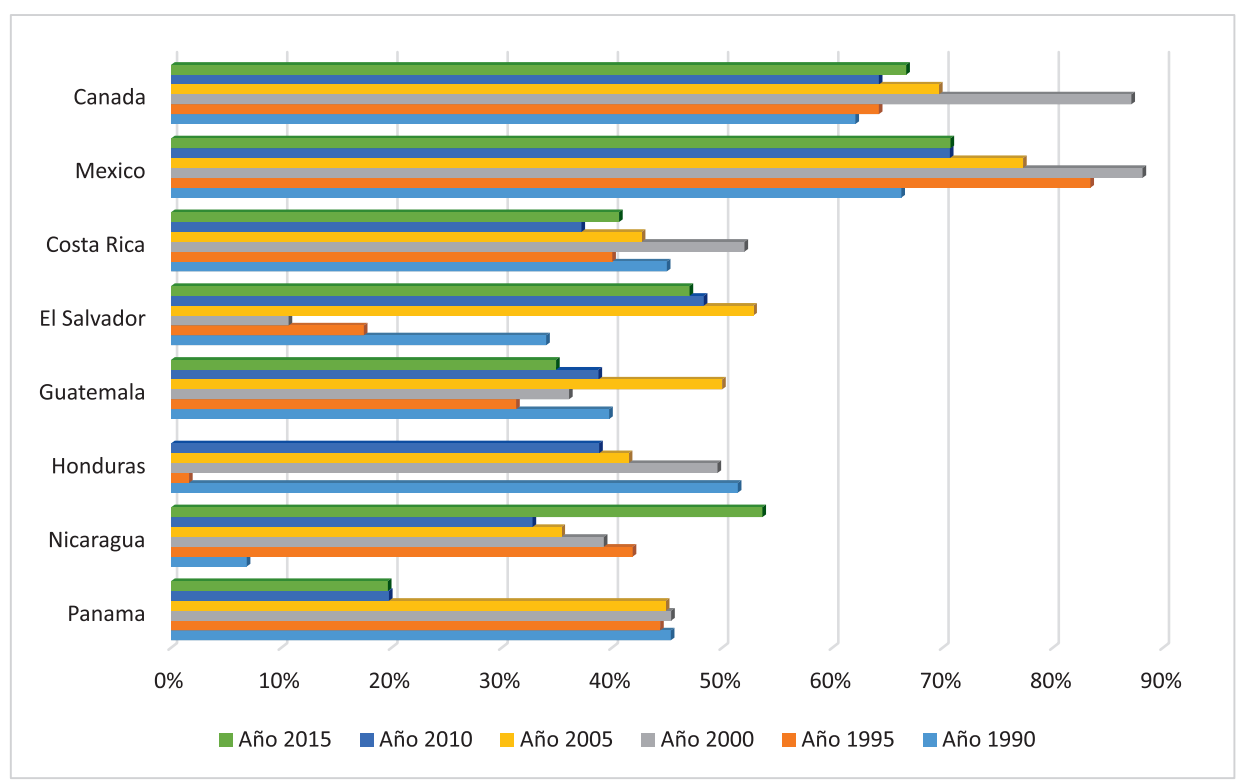

Figura 3. Estados Unidos, la economía eje en el hemisferio occidental.

Fuente: elaborado con base en datos de UNCOMTRADE (2017).

La región de Suramérica no es homogénea. Si bien existen países con índices similares a los de Centroamérica, Colombia, Ecuador y Venezuela, también están los países considerados del Cono $\operatorname{Sur}^{7}$ que muestran índices HM en niveles bajos. Así, por ejemplo, mientras Colombia a lo largo del periodo no obtuvo un índice HM inferior al $28 \%$, Paraguay presenta valores muy bajos, inferiores al $5 \%$ en los años estudiados.

Al igual que en el caso de los países de Centroamérica, se observa en la región sur una tendencia descendente en el índice HM, indudable en el caso de Bolivia, Brasil, Perú y Venezuela. Colombia, por su parte, mantiene la dependencia del mercado estadounidense, pero con una tendencia decreciente, pasando de cifras alrededor del $50 \%$ en el año 2000 hasta un $28 \%$ en el año 2015.

\section{e) Los ejes comerciales del Este Asiático y su relación con Colom- bia y los países de América}

Los análisis de los esquemas radiales de integración han identificado a Japón como el eje histórico del Este Asiático

\footnotetext{
7 El Cono Sur se plantea en este documento en un "sentido amplio". Por lo tanto, es la región integrada por Argentina, Chile, Uruguay, Bolivia, Brasil y Paraguay.
} 
y China como el nuevo eje regional, configurando la llamada "bicicleta" del Sudeste Asiático (Baldwin, 2004). Las estimaciones del índice HM para la región latinoamericana muestran una influencia importante de estos países, especialmente de la China. De esta manera, se analiza el índice HM para Japón y China (ver figuras 4 y 5). En los países del centro y norte de América la importancia del mercado japonés ha decaído a lo largo del tiempo. A comienzos de la década de los noventa, los índices
HM eran mayores en la región, superiores al $4 \%$ para Honduras, Nicaragua, Canadá y México y, superior al $9 \%$ en Estados Unidos. En 1995 empieza a descender en casi todos los países, situación que se profundiza en los quinquenios siguientes. En el último año analizado, 2015, se presenta al parecer una marginal recuperación que no compensa la caída, en países como Canadá, Costa Rica, México y Colombia. En la mayoría de países continúa siendo menor el índice con respecto al quinquenio anterior.

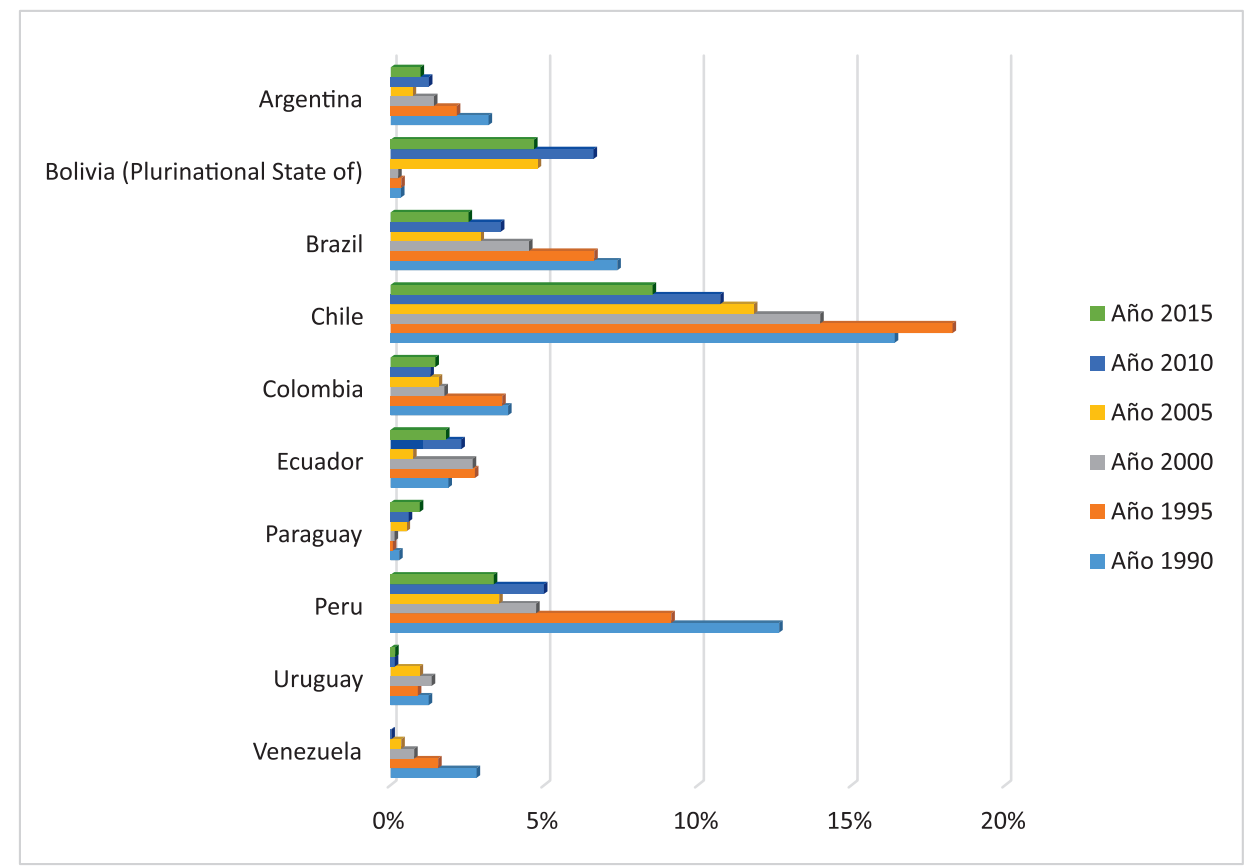




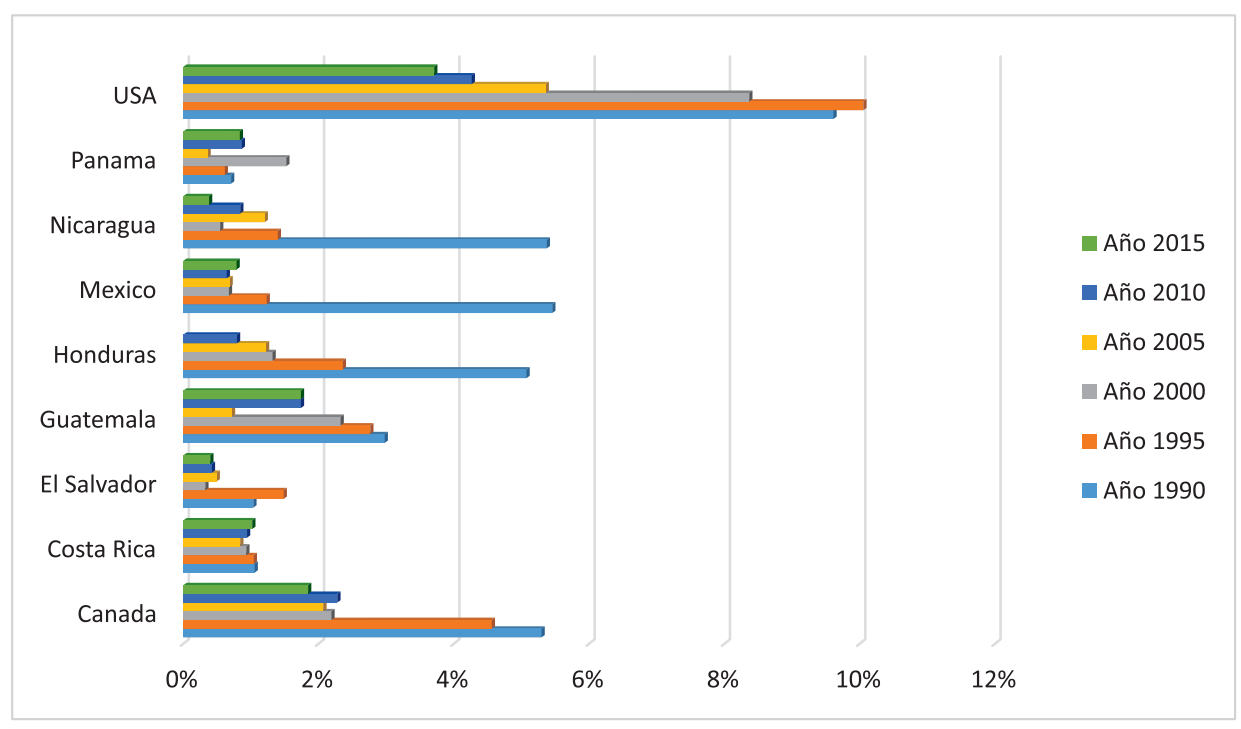

Figura 4. Japón como eje en los países de América.

Fuente: elaborado con base en datos de UNCOMTRADE (2017).

El otro eje que ha venido ganando protagonismo en los últimos años y ha alcanzado el segundo puesto dentro de los ejes más grandes en el 2015 es China (ver Figura 5). Se verifica una tendencia creciente en toda la región, respecto de la dependencia comercial frente al eje chino. Vale la pena resaltar la magnitud del índice para economías como la chilena, que supera el $26 \%$, la economía del Perú, que alcanza más del $20 \%$, la de Brasil con un $18,4 \%$, y en el caso colombiano que supera el $6 \%$.

El panorama es diferente, por los órdenes de magnitud, en la región de Norte y Centroamérica, pues el país en el que la China alcanza la mayor influencia es en los Estados Unidos con un $7 \%$, seguido de Panamá con un valor cercano al $6 \%$ y Canadá con un valor cercano al $4 \%$, y una presencia muy pequeña en Centroamérica, en términos generales. El caso de China y su creciente influencia como potencial eje en la región es llamativo, pues representa, globalmente, el paso desde una influencia comercial muy escasa hacia un aumento significativo, el cual, en el caso de Colombia, muestra una consolidación desde el año 2000 , pasando desde cifras cercanas al $1 \%$ hacia un $4 \%$. 

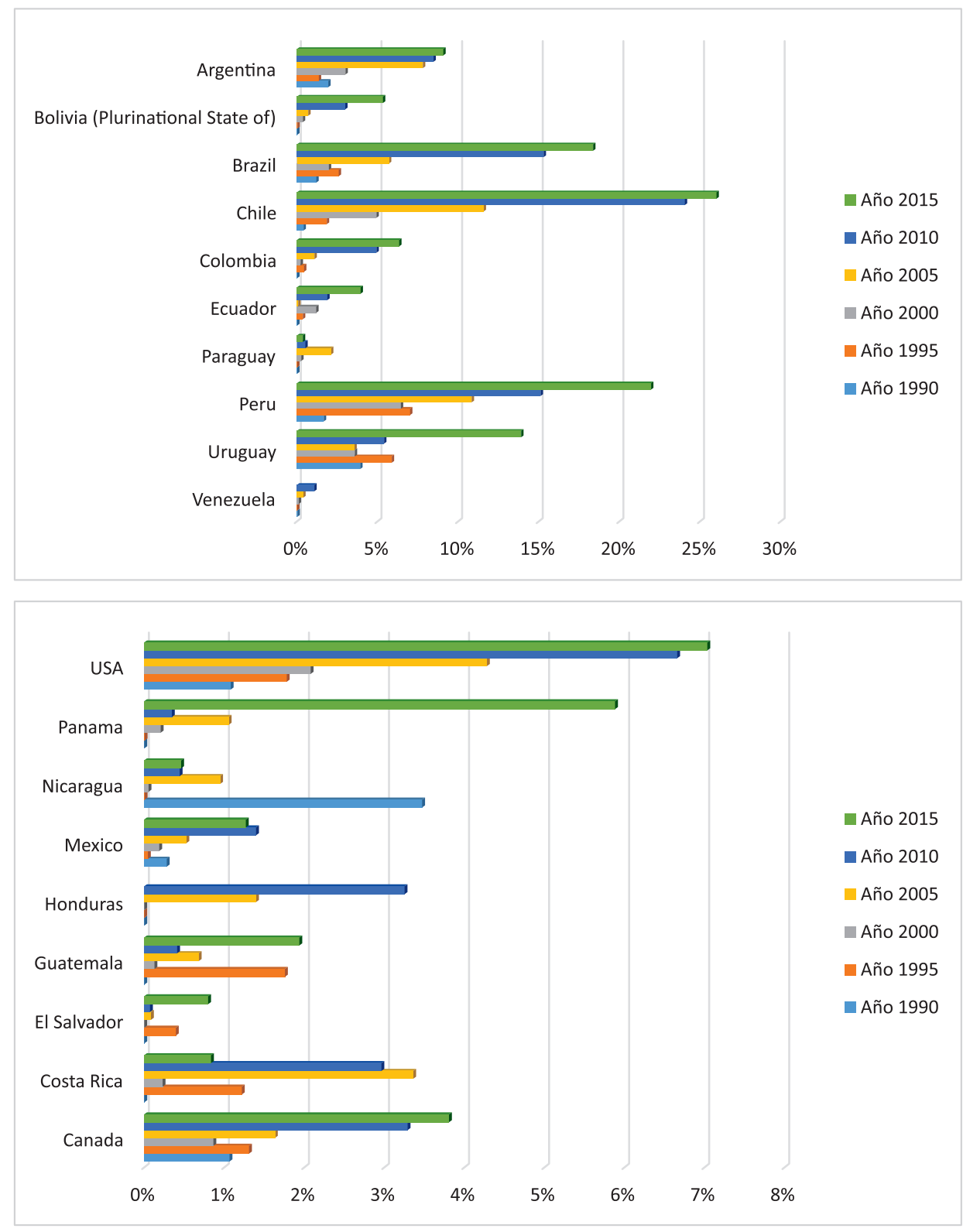

Figura 5. China como eje comercial del hemisferio.

Fuente: elaborado con base en datos de UNCOMTRADE (2017). 


\section{El fenómeno eje-radios en Co- lombia: efecto de la política de firma de acuerdos bilaterales}

Colombia ha implementado una política de suscripción de acuerdos comerciales bilaterales con varios países y continua avanzando en la consecución de nuevos acuerdos. La política de firma de acuerdos debería disminuir la dependencia comercial con los países ejes, o transformar el rol de una nación para permitirle pasar de desempeñar el rol de "radio" para ir adquiriendo el de "eje". Los resultados de la aplicación del índice HM muestran una disminución de la influencia del rol comercial de la economía de los Estados Unidos frente a la de Colombia desde el año 2000, pero también la influencia del mercado chino se ha incrementado notablemente desde ese mismo año, lo cual denota la creciente presencia de la economía de la China como candidata a convertirse en eje mundial.

Los grupos de interés en el interior de Colombia han apoyado la suscripción de acuerdos bilaterales, lo que ha llevado a configurar este patrón de eje-radios. La negociación de acuerdos comerciales bilaterales con países eje es apoyada por los exportadores, debido a que ven atractivos estos mercados, además motiva su interés la posibilidad de incrementar las exportaciones y de impedir que sean desplazados por terceros países (fortaleciendo el esquema eje-radios). Sin un esfuerzo consciente por consolidar otro rol más protagónico para la economía colombiana en el comercio internacional, el patrón de comercio eje-radios se reforzará y Colombia podría permanecer en una "trampa spoke", en su rol de economía radial. Aquí se señalan algunos de sus efectos negativos:

- La firma de acuerdos comerciales bilaterales puede reforzar la tendencia a comprar al socio comercial porque se tiene un acuerdo con él y no porque sea el más eficiente, es decir, la "desviación de comercio". Dado que el contenido de los acuerdos bilaterales no es acumulable, es probable que la desviación de comercio sea mayor, es decir, el resultado económicamente es ineficiente.

- Un acuerdo comercial bilateral puede no generar flujos comerciales reales. Así, pues, se espera que entre más acuerdos comerciales bilaterales se negocien, en especial los de última generación, los costos asociados a la implementación de mecanismos de administración y control sean mayores, debido a que cada acuerdo corresponde a un tratamiento aduanero y técnico diferente. $\mathrm{Al}$ comparar estos costos con la creación de comercio que se genera, los costos suelen ser mayores $\mathrm{y}$, por ende, el crecimiento de los flujos comerciales no es real (Lloyd \& Maclaren, 2004; Lee et al., 2005; Park \& Park, 2009). 
- El patrón eje-radios favorece al país eje a costa del país radio (Baldwin, 2004). Una de las consecuencias es que las industrias y las nuevas inversiones se ubicarán en el país eje porque el tamaño del mercado es más grande y desde allí pueden entrar al sistema de países radios. Esta concentración podría dar una ventaja al país eje, que para el país radio será difícil de superar.

- En materia política, los acuerdos comerciales bilaterales firmados pueden no ser compatibles entre sí y tienden a generar división entre países. Así, por ejemplo, el TLC con Estados Unidos y la suscripción del TLC con la Unión Europea trajo división en el interior de la CAN. También, por ejemplo, la negociación del TLC de Perú y Colombia con Estados Unidos motivó el retiro de Venezuela en 2006 y generó crisis en el interior de ese proceso de integración andina. La suscripción de TLC con grandes ejes comerciales ha fomentado, en este sentido, la división regional.

\section{CONCLUSIONES}

En el presente artículo se presenta una evaluación de la política de firma de acuerdos bilaterales de Colombia entre 1990-2015, fundamentada en un marco de análisis correspondiente al fenómeno eje-radios, lo cual permite señalar que el país está cayendo en una "trampa spoke"; es decir, que está siendo relegado a un rol secundario en el comercio internacional, en vez de ganar protagonismo, a medida que se profundiza la estrategia de proliferación de acuerdos comerciales bilaterales.

La aplicación integra dos análisis fundamentales: un análisis temporal de la evolución de Colombia frente al sistema eje-radios entre 1990-2015 usando el índice HM, y un análisis comparativo, por país eje, de Colombia frente a los demás países radios de América, por quinquenios desde 1990 a 2015, en el que la selección de los países ejes más influyentes en el mundo se hizo usando la matriz HM (Chen, 2008).

Los resultados muestran que, a pesar de hacer grandes esfuerzos en materia de política comercial centrada en la suscripción de acuerdos bilaterales, el país no puede considerarse país eje o candidato a eje para algún país radio o sistema radial de países, tampoco incluso, para países de la CAN, pues aunque se previó en algún periodo que el país ganaría protagonismo, este no logró sostenerse. Además, el índice HM para obtener los países ejes de Colombia mostró de manera clara que Estados Unidos es el país eje para Colombia a lo largo del periodo de análisis, aunque su influencia ha venido disminuyendo desde el año 2000 en favor de la influencia ganada por el eje de la China en el mismo período. 
A través de la matriz HM se obtuvo el ranking de las diez economías más importantes del mundo como ejes para cada quinquenio de 1990 hasta 2015. Estados Unidos es el país-eje más importante del mundo, ocupando el primer puesto durante el periodo de análisis. Como eje de la región, Estados Unidos continúa con la hegemonía sobre países de Centroamérica y andinos. En contraste, esta hegemonía no es tan importante para los países del Cono Sur, donde países como China y los países del interior de la región son más importantes en el comercio exterior.

Durante el periodo, China ha ganado un rol protagónico en el escenario mundial. En los años noventa y comienzos del año 2000, China no aparecía entre los diez de los ejes más grandes del mundo, pero, a partir de 2005, fue escalando posiciones, ubicándose para el 2015, en el segundo lugar de los países ejes más importantes, relegando importantes economías de la Unión Europea.

Lo anterior implica que la política de firma de acuerdos comerciales bilaterales de Colombia, a pesar de buscar que el país salga de su rol de economía radio, disminuyendo su dependencia comercial con los ejes ya menciona- dos y ganando un mayor interés de los países por el acceso preferencial a su mercado, ha generado un resultado adverso, pues se sigue reforzando el fenómeno eje-radios con Estados Unidos como eje comercial de Colombia, persistiendo su dependencia comer$\mathrm{cial}^{8}$, superior a la de otros países de Suramérica, con una influencia creciente de la economía China.

Debido a sus efectos adversos, la política comercial de firma de acuerdos a través de TLC, parece haberse convertido en un objetivo en sí misma (dada la proliferación de acuerdos), en vez de ser empleada como un instrumento (Vallejo, 2006) en el contexto más amplio de los objetivos de desarrollo económico de la nación, o como complemento de una política industrial activa. En tal sentido debe resaltarse que no cabe esperar que los TLC produzcan resultados por sí solos, sino como parte de una estrategia más amplia y coherente planteada por la nación. De no ser así, el resultado que el país obtendrá a partir de la suscripción de múltiples acuerdos comerciales bilaterales será, muy probablemente, el reforzamiento del patrón eje-radios.

Análisis como el aquí presentado contribuyen a la comprensión de las dificultades que tienen países como el


tados Unidos, es necesario que se evalúe en un horizonte temporal más amplio para que se pueda conocer su efecto sobre la relación eje-radio con Colombia.
} 
nuestro en el proceso de aprovechamiento de los acuerdos comerciales, asociado a diversos factores, relacionados con diversas modalidades de barrera comercial — por ejemplo, las normas de origen, las cuales suelen convertirse en criterios de naturaleza político-económica que representan un obstáculo técnico al comercio(Maldonado, 2014); las diversas modalidades de proteccionismo a través de la implementación de barreras no arancelarias (BNA), y diversos problemas estructurales, tales como los costos asociados al comercio exterior, el funcionamiento ineficiente de la institucionalidad y la regulación, etc. ${ }^{9}$

En último término, la presente investigación contribuye al entendimiento del fenómeno eje-radios para la política comercial de firma de acuerdos comerciales bilaterales de Colombia. En este sentido, la evaluación representa una herramienta útil para considerar una posible revisión de esta política que, hasta ahora, ha conducido a Colombia hacia una "trampa spoke".

\footnotetext{
${ }_{9}$ Los autores agradecemos a los evaluadores por hacernos notar la importancia de estos aspectos, claves en una evaluación amplia de la política comercial aplicada por nuestro país.
} 
Apuntes CENES Volumen 37, Número 65

enero - junio 2018. Págs. 117-149

\section{REFERENCIAS}

Adam, A., Kosma, T. \& McHugh, J. (2003). Trade-Liberalization Strategies: What Could Southeastern Europe Learn from the CEFTA and BFTA? IMF Working Paper 3(239), 1-31. https://doi.org/10.5089/9781451875799.001

Alba, J., Jung, H. \& Park, D. (2010). Do Hub-and-Spoke Free Trade Agreements Increase Trade? A Panel Data Analysis. ADB Working Paper Series on Regional Economic Integration 46, 1-24.

Baldwin, R. (1995). A Domino Theory of Regionalism. In Expanding Membership of the European Union (pp. 25-48). New York: Cambridge University Press. https://doi.org/10.1017/CBO9780511983368.003

Baldwin, R. (2004). The Spoke Trap: Hub and Spoke Bilateralism in East Asia. Seoul-Korea: CNAEC Research Series.

Bhagwati, J. (1995). US Trade Policy: The Infatuation with FTAs. Discussion Paper Series 726. NY: Columbia University.

Cardona, D. (2001). La política exterior de la administración Pastrana (19982000): Hacia una evaluación preliminar. Colombia Internacional, (53), 53-74.

Carrillo, C. \& Carmen, A. L. (2002). Trade Blocks and the Gravity Model: Evidence from Latin American Countries. Journal Economics Integration, 19(4), 667-689. https://doi.org/10.11130/jei.2004.19.4.667

Chen, L. (2008). The Market Driven Trade Liberalization and East Asian Regional Integration. HEID Working Paper, (12), 1-38.

Chen, L. \& Lombaerde, P. (2011). Regional Production Sharing Networks and Hub-ness in Latin America and East Asia: A Long Term-Perspective. Integration \& Trade, 15 (32), 17-34.

Chong Yuen y Hur Jung. 2008. Small Hubs, Large Spokes and Overlapping Free Trade Agreements The World Economy, 31, 1625-1665. 
Cornejo, R. \& Harris, J. (2007). Convergence in Rule of Origin Spaguetti Bowl: A Methodology Proposal. Working Paper. Washington D.C.: InterAmerican Development Bank, Trade and Integration Sector 34.

Correia, J. (2008). The Determinants of Colombian Exports: An Empirical Analysis Using the Gravity Model. Desarrollo y Sociedad, (61), 165-206.

Deltas, G., Desmet, K. \& Facchini, G. (2006). Hub-and-Spoke Free Trade Areas. Discussion paper series, (5960), 1-33.

Deltas, G., Desmet, K. \& Facchini, G. (2012). Hub-and-Spoke Free Trade Areas: Theory and Evidence from Israel. Canadian Journal of Economics, 45(3), 942-977. https://doi.org/10.1111/j.1540-5982.2012.01722.x

De Santis, R., De Benedictis, L. \& Vicarelli, C. (2005). Hub-and-Spoke or Else? Free Trade Agreements in the 'Enlarged' European Union. The European Journal of Comparative Economics, ISAE Working Paper, 2(52), 245-260.

Guacaneme, R. E. (2011). ¿Influyó la teoría de libre comercio de Mises en la apertura económica colombiana? (1990-2005). Revista Civilizar de Empresa y Economía, (3), 1-35.

Kowalczyk, C. \& Wonnacott, R.J. (1992). Hubs and Spokes, and Free Trade in the Americas. NBER Working Paper, (4198), 1-33.

Laaser, C. F. \& Schrader, K. (2002). European Integration and Changing Trade Patterns: The Case of the Baltic States. Kiel Working Paper, (1088), 1-51.

Lee, J.W., Park, I. \& Shin, K. (2008). Proliferating Regional Trade Arrangements: Why and Whither? The World Economy, 31(12), 1525-1557. https://doi. org/10.1111/j.1467-9701.2008.01143.x

Lloyd, P. \& Maclaren, D. (2004). Gains and Losses from Regional Trading Agreements. A Survey Economic Record, 80, 445-467. https://doi.org/10.1111/ j.1475-4932.2004.00202.x

Lombaerde, P. \& Garay, J.L. (2009). La economía política de la integración regional: el caso de Colombia. UNU CRIS Workig Papers, (20), 1-52. 
Maldonado, M. I. (2014). Las reglas de origen en los tratados de libre comercio. Revista de Derecho, (41), 32-50.

Martin, C. \& Turrion, J. (2001). The Trade Impact of the Integration of the CEECs on EU. European Economy Group, (11), 1-26.

Menon, J. 2008. Dealing with the Proliferation of Trade Agreements. Discussion Paper Serie APEC Study 60. NY: Columbia University.

Organización Mundial del Comercio -OMC- (2017). Los principios del sistema de comercio. Recuperado de https:/www.wto.org/spanish/thewto_s/whatis_s/tif_s/fact2_s.htm

Paas, T. (2003). Regional Integration and International Trade in the Context of EU Eastward Enlargement. HWWA Discussion Paper, (218), 1-30.

Park, I. \& Park, S. (2009). Free Trade Agreements versus Customs Unions: An Examination of East Asia. Asian Economic Paper, 8(2), 119-139. https:// doi.org/10.1162/asep.2009.8.2.119

United Nations Commodity Trade Statistics Database-UNCOMTRADE- (2017). UN Comtrade Data Base. Retrieved from https://comtrade.un.org/

Vallejo, H. (2006). El TLC andino en el contexto de Colombia. Documento CEDE, (10), 1-29.

Wonnacott, P. \& Wonnacott, R.J. (1982). Free Trade between the United States y Canada: Fifteen Years Later. Canadian Public Policy, 8, 412-427. https:// doi.org/10.2307/3549620

Wonnacott, P. \& Wonnacott, R. J. (1995). Liberalization in the Western Hemisphere: New Challenges in the Design of a Free Trade Agreement. North American Journal of Economics \& Finance, 6, 107-119. https://doi. org/10.1016/1062-9408(95)90012-8

Wonnacott, R. J. (1996). Free-Trade Agreements: For Better or Worse? The American Economic Review, 86(2), 62-66. 